Research Article

\title{
Modification of Mesoporous Silica Surface by Immobilization of Functional Groups for Controlled Drug Release
}

\author{
Abdullah M. Alswieleh \\ Chemistry Department, College of Science, King Saud University, P.O. Box 2455, Riyadh 11451, Saudi Arabia \\ Correspondence should be addressed to Abdullah M. Alswieleh; aswieleh@ksu.edu.sa
}

Received 8 April 2020; Revised 20 June 2020; Accepted 25 June 2020; Published 13 July 2020

Academic Editor: Franck Rabilloud

Copyright (C) 2020 Abdullah M. Alswieleh. This is an open access article distributed under the Creative Commons Attribution License, which permits unrestricted use, distribution, and reproduction in any medium, provided the original work is properly cited.

\begin{abstract}
This paper introduces the synthesis of mesoporous silica nanoparticles (MSNs) with three different groups such as amine, thiol, and sulfonic acid, along the internal surface. Trimethyl[3-(trimethoxysilyl)propyl]ammonium chloride was used to modify the external surface of the nanomaterials. Such materials allow control of the drug release from MSN pores. Multifunctional MSNs were loaded with doxycycline (Doxy) to study their capacities and uploading time. The loading profile indicates that sulfonic groups in the internal surface were the most efficient surfaces with a loading capacity of ca. $35 \%$ in 90 min in acidic media.
\end{abstract}

\section{Introduction}

Silica nanoparticles are prepared with different surface area, size, shape, and surface functionality. During the last decades, much attention has been paid to mesoporous nanomaterials [1-6]. Among the different silica nanoparticles, mesoporous silica nanoparticles (MSNs) are most commonly used for pharmaceutics applications due to their unique properties [4-9]. Silica surface has been modified through silane reagent attachment or coating with polymers [10-14]. Silane reagents are reacted with the surface through a condensation reaction $[15,16]$. The surface of silica can easily introduce functional groups to control drug release functions for optimized drug delivery $[17,18]$.

3-Aminopropyltriethoxysilane (APTES) and 3-mercaptopropyltrimethoxysilane (MPTMS) have been widely utilized to modify silica surface with amine and mercapto groups, respectively, and used for several applications $[17,19,20]$. Huang et al. have prepared a catalyst based on modified mesoporous silica coated with MPTMS. Thiol groups on the surface were converted to sulfonic acid and used as a catalyst for the esterification reaction [21]. Mosquera et al. reported the synthesis of gold nanoparticles stabilized with silica monolayers. The silica monolayer was formed after MPTMS immobilization on the surface of gold nanoparticles and hydrolysis in basic media [22]. Shen et al. have synthesized PEGylated capped MSNs and covered with MPTMS, to be used as a gatekeeper [23]. Cheng et al. reported the functionalization of MSNs with $\mathrm{pH}$-sensitive trimethylammonium as $\mathrm{pH}$-controllable drug release [24]. Xia et al. reported the preparation of MSNs coated with amine and encapsulated doxorubicin inside their pores and then sealed the external surface with cellulose through esterification [25]. Liu and coworkers reported the synthesis of MSNs coated with amine and used to load with doxorubicin (Dox) [26]. Chen et al. reported the preparation of mesoporous silica modified with amine for loading and releasing Dox using a capping concept. The drug can be released with control minor by detachment of the capped nanoparticles that block the MSN pores [27].

Shao et al. reported the preparation of Janus silvermesoporous silica nanoparticles (Ag-MSNs) as nanocarriers [28]. It was found that Dox-loaded Ag-MSNs were $\mathrm{pH}$ responsive drug release, leading to inhibit the growth of cancer cell, rather than human normal cells. Wang et al. engineered Janus-structured gold-mesoporous silica nanoparticles and modified with folic acid for enhancing HCC targeting and internalization [29]. Dox release from loaded nanosystem was studied from the mesopores in a $\mathrm{pH}-\mathrm{re}-$ sponsive manner. Dong and coworkers have reported the 
synthesis of Janus magnetic head with Dox-loaded mesoporous silica as "nanobullets" [30,31]. Such nanoparticles were used to inhibit the growth of cancer cell, due to $\mathrm{pH}$ promoted Dox release in the interior of cancer cells. MSNs with large internal pores have been prepared for the controlled delivery of biomacromolecules (protein and nucleic acid) with low toxicity [32].

Cheng et al. developed a multifunctional nanosystem based on Dox-gated MSNs for combined tumor chemotherapy and photothermal and gene therapies [33]. This nanoplatform could specifically target cancer cells, minimizing damage to the normal cells. A new drug delivery system based on d-a-tocopheryl polyethylene glycol 1000 succinate (TPGS)-functionalized polydopamine-coated MSNs was developed by Cheng et al. [34]. Polydopamine-coated MSNs could degrade at low $\mathrm{pH}$, leading to release Dox to damage cell lung cancer. Dual $\mathrm{pH}$ and glucose sensitivity nanocarrier were designed to control the drug release via the detection of glucose and $\mathrm{pH}$ levels [35]. Oxaliplatin prodrug (Oxa(IV)) encapsulated in TPGS functionalized MSNs was synthesized to release the drug in acidic media in cancerous tissue and the reductants in cancer cells [36]. A facile drug-selfgated MSN nanocarrier via a $\mathrm{pH}$-sensitive benzoic-imine covalent bond was demonstrated by Zeng et al. [37]. At weak acidic cancerous cells, loaded Dox could be released due to the hydrolyzation of benzoic-imine bonds. Zhang et al. fabricated bioresponsive controlled delivery nanoplatform based on the assembly and disassembly of gold nanoparticles from the MSN surface [38]. Drug could be trapped inside the nanopores by AuNPs functionalized with aptamer, and the drug can be released in the presence of adenosine molecules.

$\mathrm{Li}$ and coworkers reported the synthesis of magnetic MSNs with high drug-loading amounts and $\mathrm{pH}$-responsive control drug release. Dox-loaded magnetic MSNs could selectively damage the cancer cells in vitro [39]. Yue et al. have prepared MSNs and mesoporous organosilica nanoparticles (ss-MONs) as nanocarriers for loading Dox in liver cancer cell treatment [40]. The ssMONs-Dox showed better anticancer efficacy than MSNs-Dox, due to that ss-MONs have good degradability with reductive glutathione, leading to the enhancement of Dox release. Chen et al. have recently reported the synthesis of $\mathrm{pH} /$ glutathione (GSH) dual-sensitive nanoplatform based on silica nanoparticle for targeting cancer cells [41]. The novel nanocarrier was $\mathrm{pH}$-sensitive in the weakly acidic cancerous cells.

In this work, mesoporous silica nanoparticles (MSNs) were synthesized with amine, thiol, and sulfonic groups along the inner surface and with high surface area and average pore size of ca. $5 \mathrm{~nm}$. Thereafter, MSN external surfaces were modified with trimethyl[propyl]ammonium chloride groups. All materials were loaded with doxycycline (Doxy) to study their capacities and uploading time. Amine, thiol, and sulfonic groups in the internal surfaces may allow precise control of the drug release from MSN pores. This hypothesis is examined using BET, FT-IR, and UV-vis.

\section{Materials and Methods}

2.1. Materials. Deionized water (DI water) was taken from Elga Pure Nanopore system. Tetraethylorthosilicate (TEOS, 98\%), N-cetyltrimethylammonium bromide (CTAB, 98\%), phosphate-buffered saline (PBS), (3-aminopropyl)triethoxysilane (APTES, 95\%), ammonium hydroxide (28 wt.\%), $n$ hexane (HPLC grade), (3-mercaptopropyl)trimethoxysilane (MPTMS, 95\%), ethanol (99.8\%, HPLC grade), and toluene (analytical grade) were bought from Sigma-Aldrich. Trimethyl[3-(trimethoxysilyl)propyl]ammonium chloride (50\%) was obtained from Tokyo Chemical Industry (TCI). Ammonium nitrate (analytical grade, 95\%) was bought from Fisher Scientific. All the chemicals in this work were used as received.

\subsection{Preparation and Functionalization of Mesoporous Silica}

2.2.1. Synthesis of Mesoporous Silica Nanoparticles (MSNs). In a $250 \mathrm{ml}$ flask, CTAB ( $1 \mathrm{~g})$ was dissolved in $155 \mathrm{~mL}$ deionized water and stirred for $10 \mathrm{~min}$, and then, $\mathrm{NH}_{4} \mathrm{OH}$ $(7 \mathrm{~mL})$ was added to the mixture. A mixture of $n$-hexane $(20 \mathrm{~mL})$ and TEOS $(5 \mathrm{~mL})$ was added to the reaction under stirring and left overnight at $35^{\circ} \mathrm{C}$. Prepared nanoparticles were separated and washed with deionized water (3 times) and ethanol ( 3 times) and then dried in an oven at $110^{\circ} \mathrm{C}$.

2.2.2. Synthesis of Mesoporous Silica Nanoparticles with Amine in the Pores $\left(\mathrm{MSNs}-\mathrm{NH}_{2}\right)$. In a $250 \mathrm{ml}$ flask, CTAB $(1 \mathrm{~g})$ was dissolved in $155 \mathrm{~mL}$ deionized water and stirred for $10 \mathrm{~min}$, and then, $\mathrm{NH}_{4} \mathrm{OH}(7 \mathrm{~mL})$ was added to the mixture. A solution contained $n$-hexane $(20 \mathrm{~mL})$, APTES $(0.2 \mathrm{~mL})$, and TEOS $(5 \mathrm{~mL})$ was added to the reaction under stirring and left overnight at $35^{\circ} \mathrm{C}$. Prepared nanoparticles were separated and washed with deionized water (3 times) and ethanol (3 times) and then dried in an oven at $110^{\circ} \mathrm{C}$.

\subsubsection{Synthesis of Mesoporous Silica Nanoparticles with Thiol} in the Pores (MSNs-SH). In a $250 \mathrm{ml}$ flask, CTAB (1 g) was dissolved in $155 \mathrm{~mL}$ deionized water and stirred for $10 \mathrm{~min}$, and then, $\mathrm{NH}_{4} \mathrm{OH}(7 \mathrm{~mL})$ was added to the mixture. A solution contained $n$-hexane $(20 \mathrm{~mL})$, MPTMS $(0.2 \mathrm{~mL})$, and TEOS $(5 \mathrm{~mL})$ was added to the reaction under stirring and left overnight at $35^{\circ} \mathrm{C}$. Prepared nanoparticles were separated and washed with deionized water (3 times) and ethanol (3 times), and then, dried in an oven at $110^{\circ} \mathrm{C}$.

\subsection{Functionalization and Nanochannel Formation of Mesoporous Silica}

2.3.1. Functionalization of the External Surface of MSNs with Trimethyl[propyl]ammonium Chloride Group (TMPAC$M S N s)$. MSNs (1 g) were suspended in $40 \mathrm{~mL}$ methanol containing TMSPAC $(2 \mathrm{mmol})$. The reaction mixture was refluxed overnight (Scheme 1(a)). The product was separated by centrifugation, washed 5 times with methanol, and dried under vacuum. The template was removed using a solution 


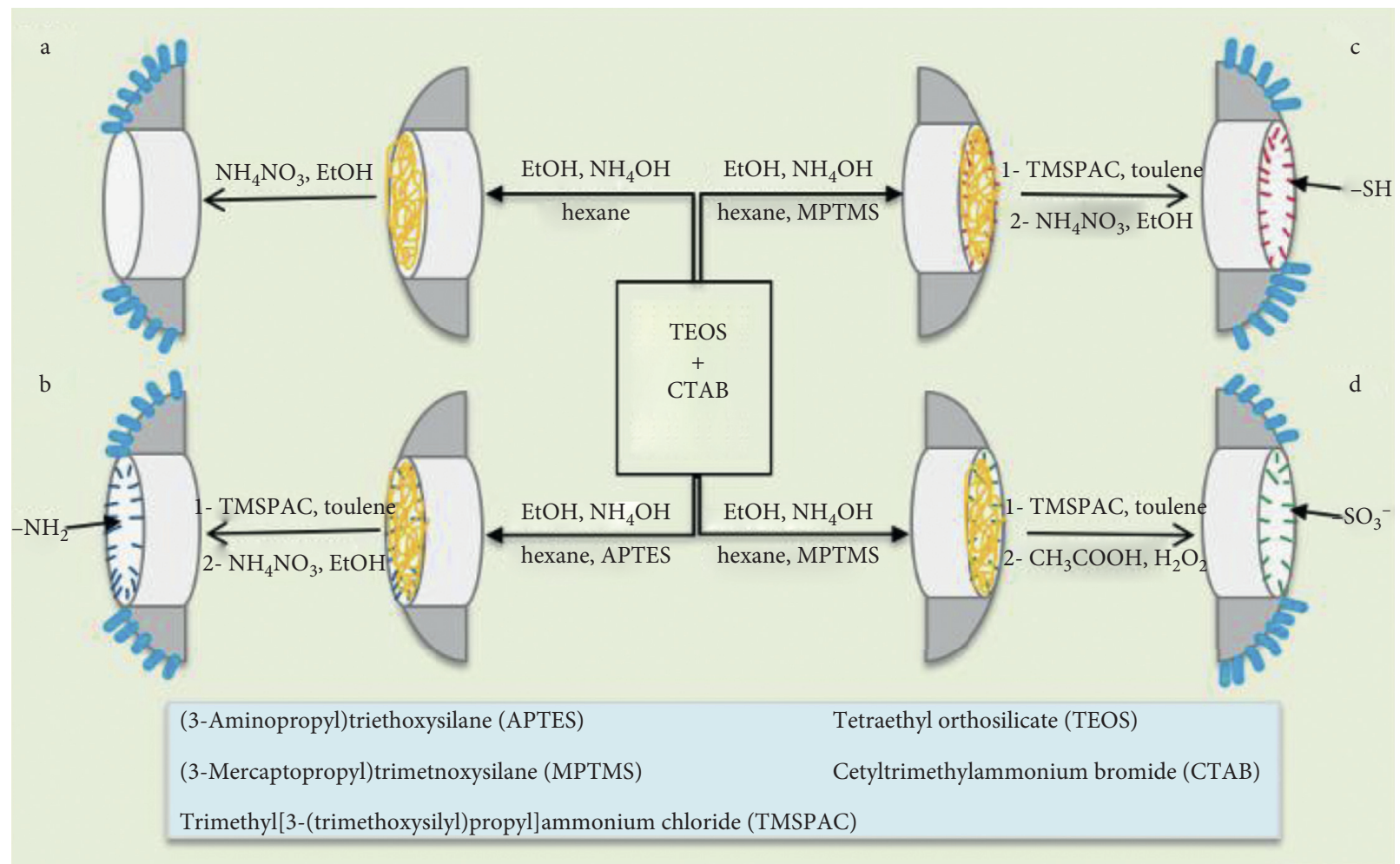

SCHEME 1: Illustration of surface modification process of mesoporous silica nanoparticles (MSNs). (a) Outer surface of MSNs modified with

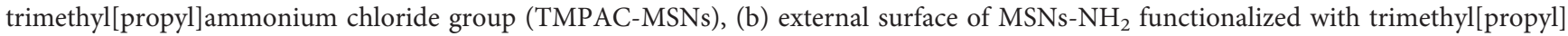
ammonium chloride group (TMPAC-MSNs- $\mathrm{NH}_{2}$ ), (c) external surface of MSNs-SH functionalized with trimethyl[propyl]ammonium chloride group (TMPAC-MSNs-SH), and (d) outer surface of $\mathrm{MSNs}^{-\mathrm{SO}_{3}^{-}}$modified with trimethyl[propyl]ammonium chloride group (TMPAC-MSNs-SO ${ }_{3}^{-}$).

of ammonium nitrate in ethanol $(10 \mathrm{mg} / \mathrm{mL})$ at $85^{\circ} \mathrm{C}$ for $12 \mathrm{~h}$ under stirring. The nanoparticles were centrifuged, washed 5 times with ethanol, and dried in an oven.

2.3.2. Functionalization of the External Surface of MSNs$\mathrm{NH}_{2}$ with Trimethyl[propyl]ammonium Chloride Group (TMPAC-MSNs- $\mathrm{NH}_{2}$ ). $1 \mathrm{~g}$ of $\mathrm{MSNs}-\mathrm{NH}_{2}$ was suspended in $40 \mathrm{~mL}$ methanol containing TMSPAC $(2 \mathrm{mmol})$. The reaction mixture was refluxed overnight (Scheme 1(b)). The product was separated by centrifugation, washed 5 times with methanol, and dried under vacuum. The template was removed using a solution of ammonium nitrate in ethanol $(10 \mathrm{mg} / \mathrm{mL})$ at $85^{\circ} \mathrm{C}$ for $12 \mathrm{~h}$ under stirring. The nanoparticles were centrifuged, washed 5 times with ethanol, and dried in an oven.

\subsubsection{Functionalization of the External Surface of MSNs-SH with Trimethyl[propyl]ammonium Chloride Group} (TMPAC-MSNs-SH). $1 \mathrm{~g}$ of MSNs-SH was suspended in $40 \mathrm{~mL}$ methanol containing TMSPAC $(2 \mathrm{mmol})$. The reaction mixture was refluxed overnight (Scheme 1(c)). The product was separated by centrifugation, washed 5 times with methanol, and dried under vacuum. The template was removed using a solution of ammonium nitrate in ethanol $(10 \mathrm{mg} / \mathrm{mL})$ at $85^{\circ} \mathrm{C}$ for $12 \mathrm{~h}$ under stirring. The nanoparticles were centrifuged, washed 5 times with ethanol, and dried in an oven.

\subsubsection{Functionalization of the External Surface of $\mathrm{MSNs}_{-} \mathrm{SO}_{3}^{-}$ with Trimethyl[propyl]ammonium Chloride Group} (TMPAC-MSNs (-SO $\mathrm{S}_{3}^{-}$in Inner Surface)). $1 \mathrm{~g}$ of MSNs-SH was suspended in $40 \mathrm{~mL}$ methanol containing TMSPAC $(2 \mathrm{mmol})$. The reaction mixture was refluxed overnight (Scheme 1(d)). The product was separated by centrifugation, washed 5 times with methanol, and dried under vacuum. The template was removed using a mixture of acetic acid and hydrogen peroxide under refluxing for $12 \mathrm{~h}$ under stirring. The final product was centrifuged, washed with ethanol, and dried in an oven.

2.4. Doxycycline (Doxy) Loading and pH-Triggered Release. To determine the loading capacity and efficiency of encapsulation at different times, each prepared nanoparticle was dispersed in deionized water with a concentration of $1 \mathrm{mg} / \mathrm{mL}$. Doxy was added to the suspension with a concentration of $0.5 \mathrm{mg}$ Doxy per $\mathrm{mg}$ of fabricated nanoparticles, and the $\mathrm{pH}$ of the resulting mixture was adjusted to 6.5 or 7.4. Each mixture was shaken at room temperature for $5,10,20,30,40,60,120,180,240,360$, or $480 \mathrm{~min}$. After each time period, the mixtures were centrifuged and washed two times with $1 \mathrm{~cm}^{3}$ buffer solutions. All fragments of each 
experiment were combined to determine the Doxy concentration in the final solution by the colorimetric method. The unloaded Doxy concentration was calculated on the basis of a calibration curve of Doxy in buffer.

Doxy-loaded nanoparticles of each type were suspended in $(\mathrm{pH}=3, \mathrm{pH}=5, \mathrm{pH}=6.5, \mathrm{pH}=7.4$, and $\mathrm{pH}=8.5)$ solution at a concentration of $0.25 \mathrm{mg}$ NPs/mL. At a certain time, the nanoparticles were separated, and aliquot supernatant was removed and replaced with an equal volume of buffer solution. The amount of Doxy released was determined based on the calibration curve.

The efficiency of encapsulation (EE) and loading capacity (LC) was calculated as follows:

$$
\begin{aligned}
& \mathrm{LC}(\%)=\left(\frac{\text { weight of loaded drug }}{\text { weight of nanoparticles }}\right) \times 100, \\
& \mathrm{EE}(\%)=\left(\frac{\text { weight of loaded drug }}{\text { weight of drug in feed }}\right) \times 100 .
\end{aligned}
$$

2.5. Measurement and Characterization. Scanning electron microscopy (SEM): JEOL JSM-6380 LA was used to image the dried nanomaterial without any treatment. Transmission electron microscopy (TEM): a drop of dilute nanoparticles suspended in HPLC ethanol was placed on a copper grid and dried at $25^{\circ} \mathrm{C}$, and then, TEM image was collected by JEOL JEM-1230. Surface area analysis: nanoparticle surface areas were determined by nitrogen physisorption isotherms on a Micromeritics Gemini 2375 volumetric analyzer. Each prepared sample was degassed at least $5 \mathrm{~h}$ at $120^{\circ} \mathrm{C}$. The Brunauer-Emmett-Teller (BET) surface areas were calculated by experimental points at a relative pressure $\left(P / P^{\circ}\right)$ of $0.05-0.25$. The pore volume was calculated from the adsorption of $\mathrm{N}_{2}$ at the $P / P^{\circ}$ of 0.99 . The average pore size distribution was determined using the Barrett-Joyner-Halenda (BJH) model. FTIR spectroscopy: IR spectra of all prepared samples were collected by the Thermo Scientific Nicolet iS10 instrument. The spectra were obtained in $\mathrm{KBr}$ pellets in the $4000-400 \mathrm{~cm}^{-1}$ region with a resolution of $4 \mathrm{~cm}^{-1}$. UV-vis spectra: Shimadzu (UV-2600) UV-vis spectrophotometer was used for all experiments. Dynamic light scattering (DLS): the intensity-average hydrodynamic diameter of all nanomaterials was measured by the Malvern Zetasizer instrument. All samples were dispersed in a buffer solution of PBS and analyzed using disposable plastic cuvettes.

\section{Results and Discussion}

Scanning electron microscopy (SEM) and transmission electron microscopy (TEM) were utilized to study the morphology of the prepared samples. SEM image shows that mesoporous silica is nearly spherical nanoparticle, with an average particle size of ca. $200 \mathrm{~nm}$, as shown in Figure 1(a). TEM image reveals that prepared samples are dispersed without aggregation, with a diameter ranging from 150 to $250 \mathrm{~nm}$, which shows a good agreement with the SEM image (Figure 1(b)). Highly ordered array pores were observed from the TEM image, with a pore size of ca. $5 \mathrm{~nm}$. No significant differences in SEM and TEM images were observed between nonmodified and modified MSNs, which is similar to the observation reported previously [42] (Figures 1S and 2S). Furthermore, the particle size distribution was investigated using dynamic light scattering (DLS). Although some particles had sizes around $400 \mathrm{~nm}$, the majority ranged between 100 and $300 \mathrm{~nm}$. The average particle size was $220 \mathrm{~nm}$ for all fabricated nanomaterials (Figure 3S). This shows a good agreement with the size estimated by SEM and TEM.

Bare MSNs, TMPAC-MSNs, TMPAC-MSNs- $\mathrm{NH}_{2}$, TMPAC-MSNs-SH, and TMPAC-MSNs-SO - were characterized using Brunauer-Emmett-Teller (BET). The $\mathrm{N}_{2}$ adsorption/desorption isotherms were found to be type IV for all samples, which are typically for the mesoporous materials (Figure 2). At higher relative pressures, small different capillary condensation steps were observed for TMPAC-MSNs- $\mathrm{NH}_{2}$, TMPAC-MSNs-SH, and TMPACMSNs-SO ${ }_{3}^{-}$compared with bare MSNs and TMPAC-MSNs. The hysteresis loop was broader for bare MSNs and TMPAC-MSNs, compared to MSN internal modified surface, which confirms the modification process.

The physicochemical properties of all the samples are presented in Table 1. Generally, there were reductions in surface area, and pore volumes were noted for functionalized MSNs. The average pore size was between 5 and $6 \mathrm{~nm}$ for all prepared samples, which agrees with the pore size that observed in the TEM image.

FT-IR was utilized to characterize the bare MSNs, TMPAC-MSNs, TMPAC-MSNs-NH 2 , TMPAC-MSNs-SH, and TMPAC-MSNs-SO $\mathrm{S}_{3}^{-}$, as shown in Figure 3. It can be seen that wide bands at $1250-1050 \mathrm{~cm}^{-1}$ were ascribed to siloxane groups ( $\mathrm{Si}-\mathrm{O}-\mathrm{Si}$ ) bands, and peak at $800 \mathrm{~cm}^{-1}$ was ascribed to $\mathrm{Si}-\mathrm{O}$ stretching vibration. Peak was noticed in all prepared samples at $1650 \mathrm{~cm}^{-1}$, which was ascribed to water bending vibration. The absorption peaks of surface-modified nanoparticles were noted at $1490 \mathrm{~cm}^{-1}$, which were ascribed to $\mathrm{C}-\mathrm{H}$ as bending vibration in $-\mathrm{CH}_{2}-$ groups, and peaks around $2930 \mathrm{~cm}^{-1}$ were assigned to $\mathrm{C}-\mathrm{H}$ as stretch vibration. These characters provide an evidence of the successful functionalization of surface MSNs.

Elemental analysis was used to estimate the molecules attached to the surfaces of bare MSNs, TMPAC-MSNs, MSNs- $\mathrm{NH}_{2}$, TMPAC-MSNs- $\mathrm{NH}_{2}$, MSNs-SH, TMPACMSNs-SH, MSNs-SO - , and TMPAC-MSNs-SO ${ }_{3}^{-}$using the measured percentages of carbon, hydrogen, nitrogen, and sulfur (Table 2). The results clearly show an increase in the percentage of N, C, S, and H elements on the modified MSNs compared to bare MSNs, which confirms the presence of the functional groups in internal and external surfaces of MSNs.

Doxy is a tetracycline antibiotic that is widely used to treat serious bacterial infections, such as cholera, typhus, and syphilis. To the best of our knowledge, little work focuses on the interaction between Doxy and the internal surface of mesoporous silica nanoparticles. The main goal of this study is to investigate the effect of selected functional groups in the inner surface on the drug release. To minimize the external surface effect, the outer surface of all fabricated nanoparticles was functionalized with TMPAC. Loading and 


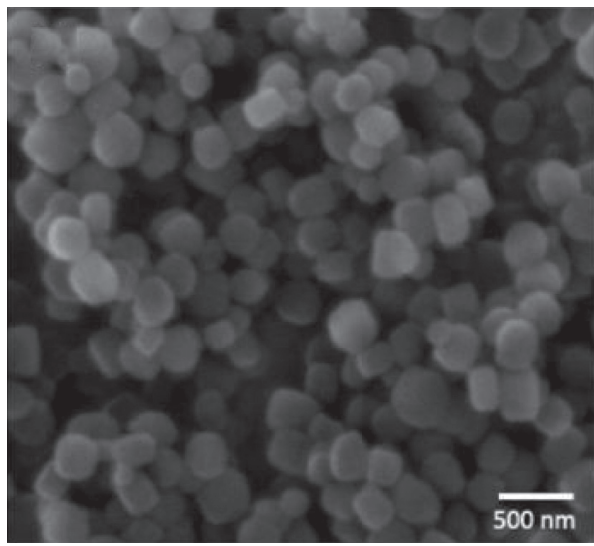

(a)

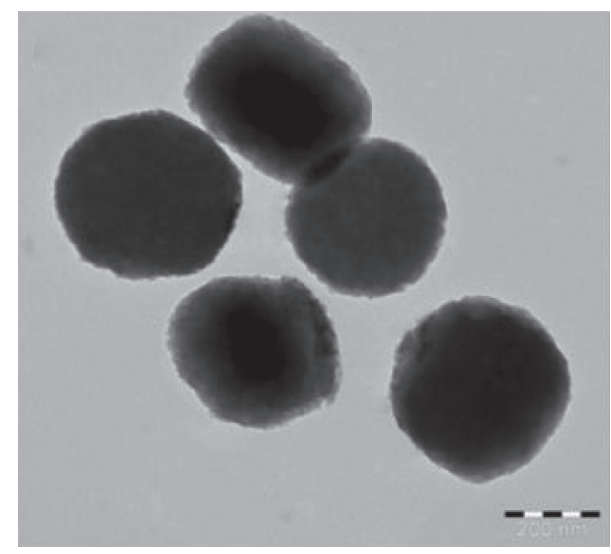

(b)

FIgURE 1: (a) SEM images of fabricated mesoporous silica nanoparticles (bare MSNs). (b) TEM images of prepared mesoporous silica nanoparticles (bare MSNs).

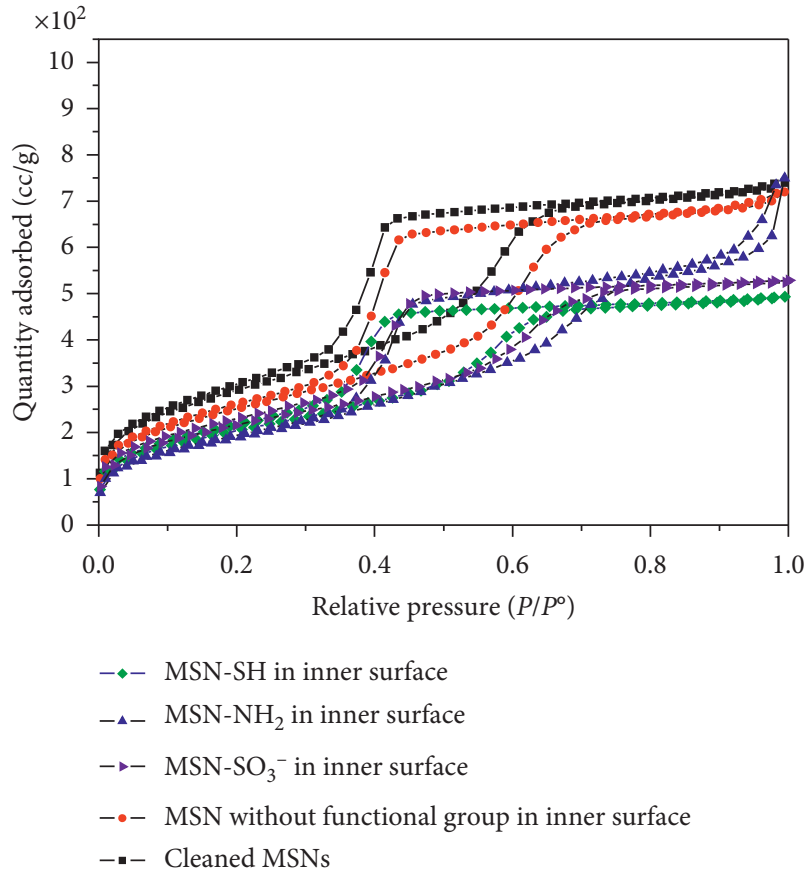

FIgURE 2: Nitrogen adsorption isotherms of ( $\boldsymbol{\square}$ black) bare MSNs, $\left(\bullet\right.$ red) TMPAC-MSNs, ( $\boldsymbol{\Delta}$ blue) TMPAC-MSNs $-\mathrm{NH}_{2}$, ( $\bullet$ green) TMPAC-MSNs-SH, and ( $\mathbf{\square}$ purple) TMPAC-MSNs-SO ${ }_{3}^{-}$.

TABle 1: Physiochemical data obtained for fabricated nanoparticles.

\begin{tabular}{lcc}
\hline Sample ID & $\begin{array}{r}\text { BET surface area } \\
\left(\mathrm{m}^{2} \cdot \mathrm{g}^{-1}\right)\end{array}$ & $\begin{array}{c}\text { Pore volume } \\
\left(\mathrm{cm}^{3} \cdot \mathrm{g}^{-1}\right)\end{array}$ \\
\hline Bare MSNs & 1029 & 1.18 \\
TMPAC-MSNs & 875 & 1.08 \\
TMPAC-MSNs- & 692 & 0.88 \\
$\mathrm{NH}_{2}$ & 709 & 0.79 \\
TMPAC- & & \\
MSNs-SH & 718 & 0.83 \\
TMPAC- & & \\
MSNs-SO &
\end{tabular}

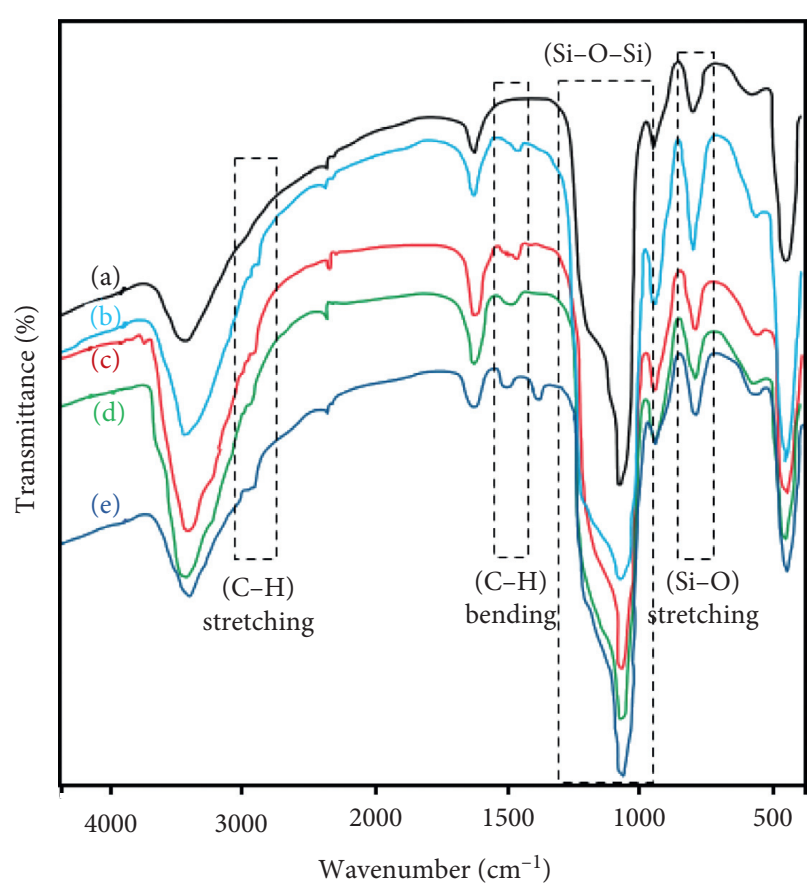

Figure 3: FT-IR spectra obtained for (a) bare MSNs, (b) TMPACMSNs, (c) TMPAC-MSNs- $\mathrm{NH}_{2}$, (d) TMPAC-MSNs-SH, and (e) TMPAC-MSNs-SO ${ }_{3}^{-}$.

TABLE 2: Elemental analysis data obtained for the fabricated mesoporous silica nanoparticles.

\begin{tabular}{|c|c|c|c|c|}
\hline Sample ID & $\mathrm{N}(\%)$ & $\mathrm{C}(\%)$ & $\mathrm{H}(\%)$ & S (\%) \\
\hline Bare MSNs & - & 1.41 & - & - \\
\hline TMPAC-MSNs & 1.14 & 6.51 & 1.78 & - \\
\hline MSNs- $\mathrm{NH}_{2}$ & 0.83 & 5.39 & 1.43 & - \\
\hline TMPAC-MSNs- $\mathrm{NH}_{2}$ & 1.79 & 8.71 & 2.17 & - \\
\hline MSNs-SH & - & 4.49 & 1.31 & 2.09 \\
\hline TMPAC-MSNs-SH & 1.24 & 7.82 & 2.14 & 1.82 \\
\hline MSNs-SO ${ }_{3}^{-}$ & - & 4.19 & 1.28 & 1.41 \\
\hline TMPAC-MSNs-SO & 1.13 & 8.14 & 2.20 & 1.87 \\
\hline
\end{tabular}






(a)

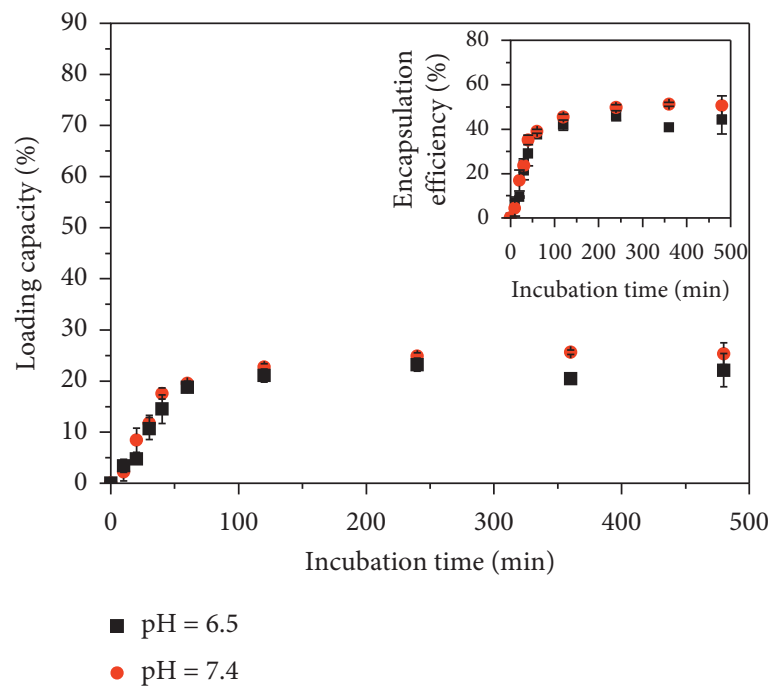

(c)

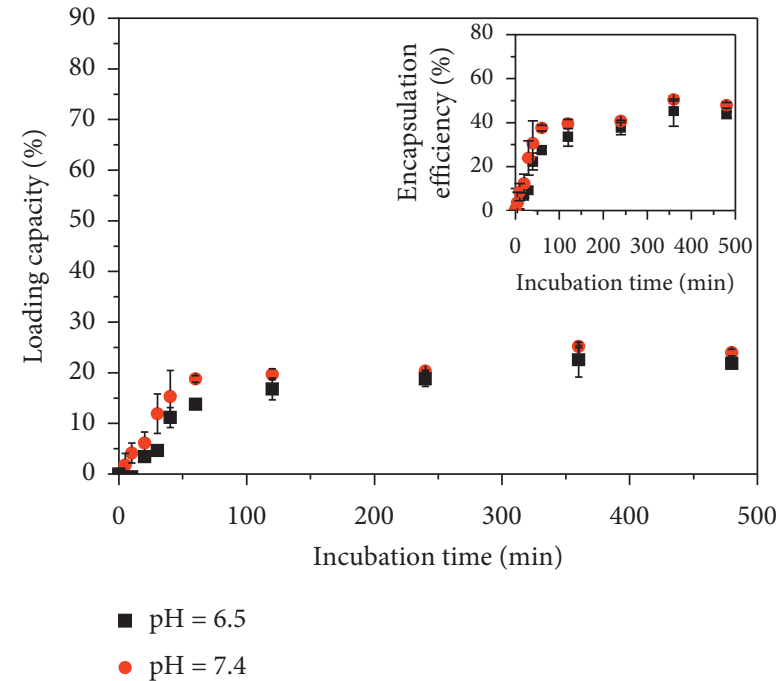

(b)

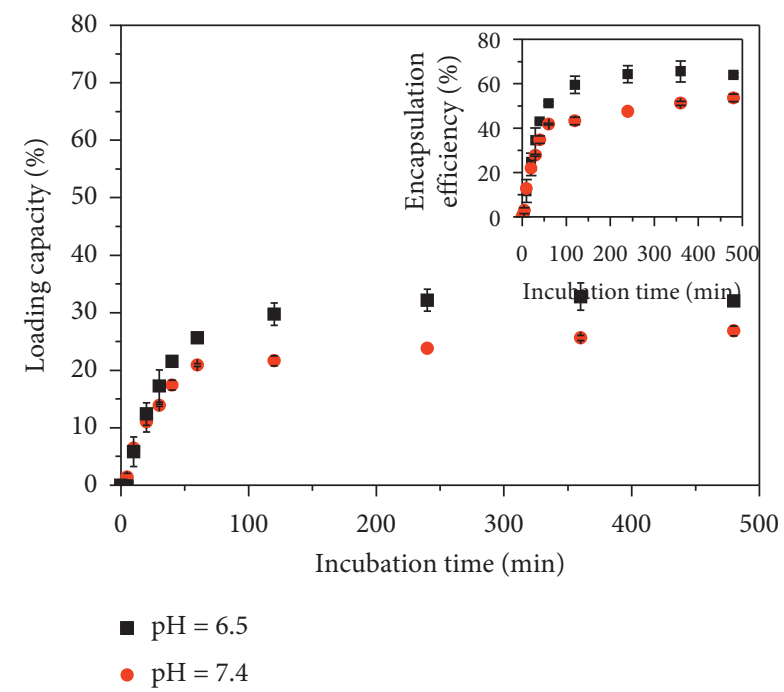

(d)

FIgure 4: Drug-loading profiles of Doxy in nanocarriers at different $\mathrm{pH}$ and loading time: (a) TMPAC-MSNs, (b) TMPAC-MSNs-NH${ }_{2}$, (c) TMPAC-MSNs-SH, and (d) TMPAC-MSNs-SO - .

release of Doxy were investigated as drug delivery vectors for TMPAC-MSNs, TMPAC-MSNs-NH $\mathrm{N}_{2}$, TMPAC-MSNs-SH, and TMPAC-MSNs-SO- $\mathrm{S}_{3}^{-}$. Doxy was loaded in these different MSNs samples at $\mathrm{pH}$ of 6.5 and 7.4 and at different loading time to allow Doxy to interact with the inner surfaces of the MSNs (Figure 4). For TMPAC-MSNs, TMPAC-MSNs- $\mathrm{NH}_{2}$, and TMPAC-MSNs-SH, the loading capacity dramatically increased to ca. $20 \%$, in $60 \mathrm{~min}$, and then remains at $25 \%$, after $90 \mathrm{~min}$ for TMPAC-MSNs and TMPAC-MSNs- $\mathrm{NH}_{2}$ at both $\mathrm{pH} 6.5$ and 74 . However, in $60 \mathrm{~min}$, the loading capacity of TMPAC-MSNs-SO- increased to ca. $20 \%$ at $\mathrm{pH} 7.4$ and $30 \%$ at $\mathrm{pH} 6.5$ and then remains at $35 \%$, after $90 \mathrm{~min}$. The enhanced encapsulation efficiency of TMPAC-MSNs$\mathrm{SO}_{3}^{-}$compared to other fabricated materials indicates that Doxy (positive) could interact physically to the interior surfaces of TMPAC-MSNs-SO ${ }_{3}^{-}$. The encapsulation efficiency for all fabricated materials was ca. $40 \%$ at different
$\mathrm{pH}$, except TMPAC-MSNs-SO ${ }_{3}^{-}$. The encapsulation efficiency of MSNs with sulfonic groups in the internal surface was ca. $60 \%$ in acidic media. The surface charge of all prepared nanoparticles was found to be around $40 \mathrm{mV}$ at $\mathrm{pH}$ 7.4 , due to the surface functionalization with TMPAC (permanent positively charged) on the external surface of MSNs. It was noted that Doxy-loaded MSNs had similar surface zeta potential of unloaded MSNs (Figure 4S).

The Doxy release profile of the fabricated nanoparticles was investigated in mild acidic ( 3,5 , and 6.5$)$ and basic (7.4 and 8.5) $\mathrm{pH}$ conditions at room temperature (Figure 5). The unmodified inner surfaces of MSNs material present clear drug release behaviour (Figure 5(a)). At pH higher than 5, about $20 \%$ release was noticed after an incubation of 72 hours. The drug release was higher at $\mathrm{pH} 3$, to ca. $25 \%$, which may be assigned to the physical adsorption between the drug and the inner surfaces of the samples. The release profile of 

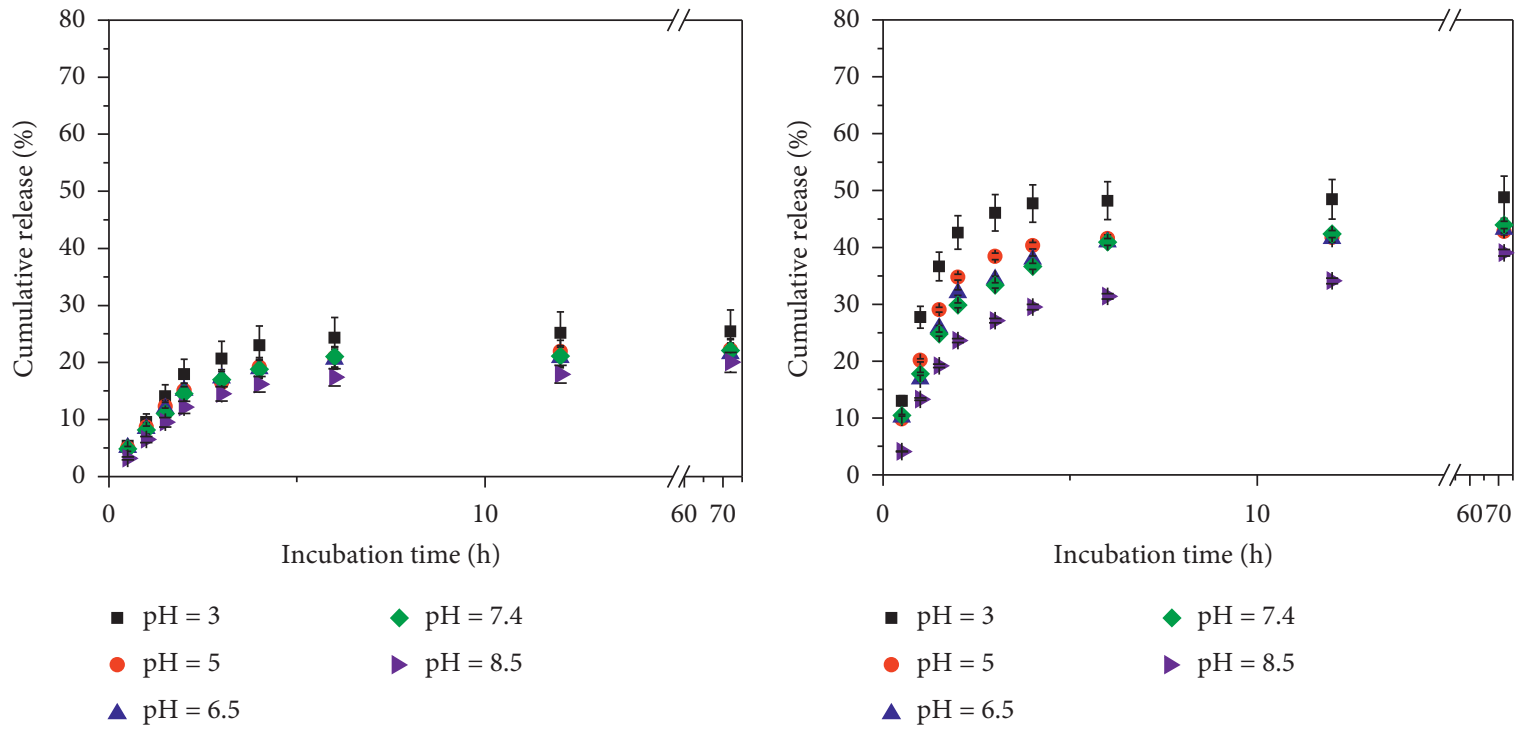

(a)

(b)

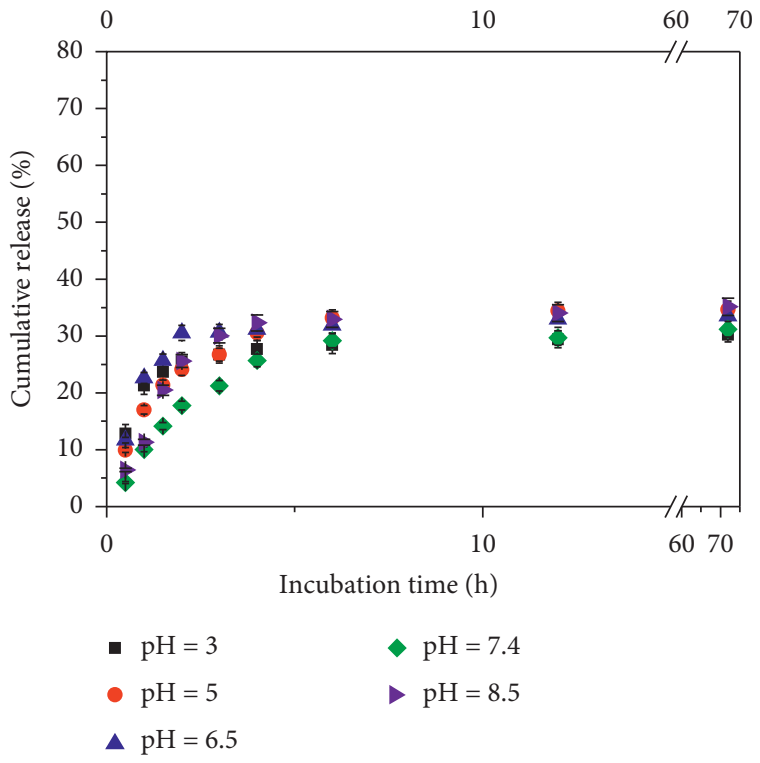

(c)

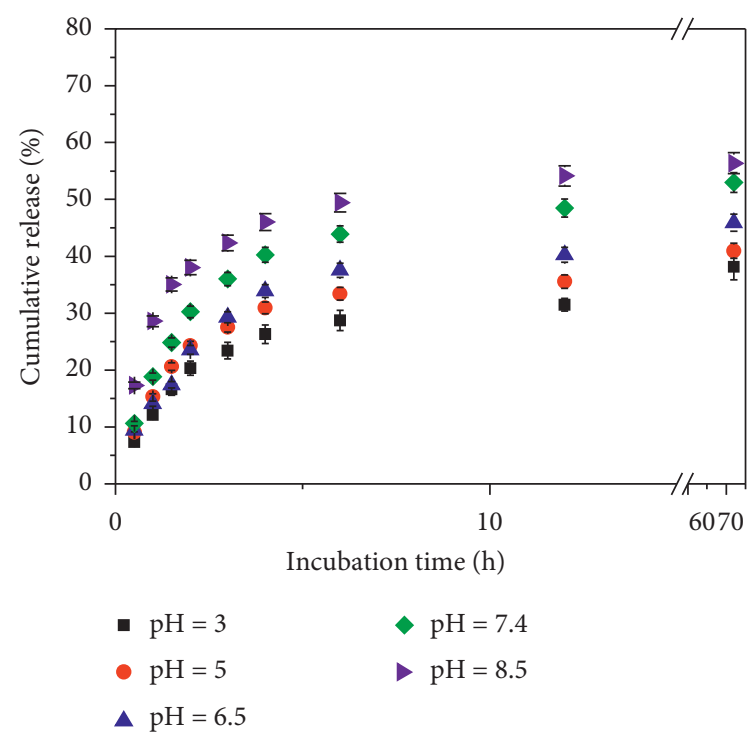

(d)

Figure 5: Drug release profiles of Doxy in nanocarriers at different $\mathrm{pH}$ and loading time: (a) TMPAC-MSNs, (b) TMPAC-MSNs-NH${ }_{2}$, (c) TMPAC-MSNs-SH, and (d) TMPAC-MSNs-SO . $^{-}$

TMPAC-MSNs- $\mathrm{NH}_{2}$ exhibited a constant release of ca. $35 \%$ at $\mathrm{pH} 8.5$, after a 72 -hour incubation period (Figure 5(b)). Upon decreasing the $\mathrm{pH}$ to $5,6.5$, and 7.4, approximately $40 \%$ of Doxy was released after a 72 -hour incubation period. However, almost $50 \%$ of the drug was released when the Doxy-loaded TMPAC-MSNs- $\mathrm{NH}_{2}$ exposed to $\mathrm{pH}$ 3. The observation may be assigned to the attractive and repelling forces between the amino-modified internal surface and Doxy. The Doxy release profile of TMPAC-MSNs-SH is presented in Figure 5(c). No difference in the release behaviour was observed when the $\mathrm{pH}$ was changed. This results that the interaction behaviour between the thiol group and Doxy is not affected by the $\mathrm{pH}$. Yet, when the thiol group was converted to sulfonic groups, Doxy release was ca. 55\% after
72 hours at $\mathrm{pH} 7.4$ and 8.5. In acidic media, there was a reduction in Doxy release due to the physical interaction between the surface and the drug.

\section{Conclusions}

In summary, we reported the preparation of MSNs different functional groups in the internal surface, and high surface area of $\sim 1000 \mathrm{~m}^{2} / \mathrm{g}$, large pore volume of $1.2 \mathrm{~cm}^{3} / \mathrm{g}$, and average pore size of $\sim 5.5 \mathrm{~nm}$. Such materials were covered with cationic groups by attaching trimethyl[3-(trimethoxysilyl)propyl]ammonium chloride in the external surface. The characterizations via TEM, FT-IR, BET, and elemental analysis confirmed that the MSNs were prepared 
successfully. The loading capacity of TMPAC-MSNs-SO was found to be the highest with $30 \%$ at $\mathrm{pH}$ 6.5. Furthermore, the drug release was ca. 55\% in a relatively controlled manner.

\section{Data Availability}

All data used to support the findings of the study are available within the article and from the corresponding author.

\section{Conflicts of Interest}

The author declares no conflicts of interest.

\section{Acknowledgments}

The author extends this appreciation to the Deanship of Scientific Research at King Saud University for funding this work through the research project.

\section{Supplementary Materials}

Figure 1S: SEM images of (A) TAMPAC-MSNs-NH2, (B) TAMPAC-MSNs-SH, and (C) TAMPAC-MSNs- $\mathrm{SO}_{3}{ }^{-}$. Figure 2S: TEM images of (A) TAMPAC-MSNs-NH2, (B) TAMPAC-MSNs-SH, and (C) TAMPAC-MSNs- $\mathrm{SO}_{3}{ }^{-}$. Figure 3S: size distribution of fabricated MSNs in PBS at $\mathrm{pH}$ 7.4. Figure 4S: surface zeta potential of unloaded and Doxyloaded MSNs in PBS at pH 7.4. (Supplementary Materials)

\section{References}

[1] J. S. Beck, J. C. Vartuli, W. J. Roth et al., "A new family of mesoporous molecular sieves prepared with liquid crystal templates," Journal of the American Chemical Society, vol. 114, no. 27, pp. 10834-10843, 1992.

[2] S.-H. Wu, C.-Y. Mou, and H.-P. Lin, "Synthesis of mesoporous silica nanoparticles," Chemical Society Reviews, vol. 42, no. 9, pp. 3862-3875, 2013.

[3] S.-J. Reich, A. Svidrytski, A. Höltzel et al., "Hindered diffusion in ordered mesoporous silicas: insights from pore-scale simulations in physical reconstructions of SBA-15 and KIT-6 silica," The Journal of Physical Chemistry C, vol. 122, no. 23, pp. 12350-12361, 2018.

[4] N. K. Mal, M. Fujiwara, and Y. Tanaka, "Photocontrolled reversible release of guest molecules from coumarin-modified mesoporous silica," Nature, vol. 421, no. 6921, pp. 350-353, 2003.

[5] S. L. Burkett, S. D. Sims, and S. Mann, "Synthesis of hybrid inorganic-organic mesoporous silica by co-condensation of siloxane and organosiloxane precursors," Chemical Communications, no. 11, pp. 1367-1368, 1996.

[6] P. Singh, S. Srivastava, and S. K. Singh, "Nanosilica: recent progress in synthesis, functionalization, biocompatibility, and biomedical applications," ACS Biomaterials Science \& Engineering, vol. 5, no. 10, pp. 4882-4898, 2019.

[7] J. Lu, M. Liong, Z. Li, J. I. Zink, and F. Tamanoi, "Biocompatibility, biodistribution, and drug-delivery efficiency of mesoporous silica nanoparticles for cancer therapy in animals," Small, vol. 6, no. 16, pp. 1794-1805, 2010.
[8] K.-J. Chen, H.-L. Chen, C.-C. Tang, H.-H. Wu, and J.-S. Jan, "Synthesis of silica/polypeptide hybrid nanomaterials and mesoporous silica by molecular replication of sheet-like polypeptide complexes through biomimetic mineralization," Journal of Colloid and Interface Science, vol. 542, pp. 243-252, 2019.

[9] F. Tang, L. Li, and D. Chen, "Mesoporous silica nanoparticles: synthesis, biocompatibility and drug delivery," Advanced Materials, vol. 24, no. 12, pp. 1504-1534, 2012.

[10] I. I. Slowing, J. L. Vivero-Escoto, B. G. Trewyn, and V. S.-Y. Lin, "Mesoporous silica nanoparticles: structural design and applications," Journal of Materials Chemistry, vol. 20, no. 37, pp. 7924-7937, 2010.

[11] C. Liu, J. Guo, W. Yang, J. Hu, C. Wang, and S. Fu, "Magnetic mesoporous silica microspheres with thermo-sensitive polymer shell for controlled drug release," Journal of Materials Chemistry, vol. 19, no. 27, pp. 4764-4770, 2009.

[12] Y. Wang, M. Yin, X. Lin et al., "Tailored synthesis of polymerbrush-grafted mesoporous silicas with $\mathrm{N}$-halamine and quaternary ammonium groups for antimicrobial applications," Journal of Colloid and Interface Science, vol. 533, pp. 604-611, 2019.

[13] R. Liu, P. Liao, J. Liu, and P. Feng, "Responsive polymercoated mesoporous silica as a $\mathrm{pH}$-sensitive nanocarrier for controlled release," Langmuir, vol. 27, no. 6, pp. 3095-3099, 2011.

[14] X. Zhang, P. Yang, Y. Dai et al., "Multifunctional up-converting nanocomposites with smart polymer brushes gated mesopores for cell imaging and thermo/pH dual-responsive drug controlled release," Advanced Functional Materials, vol. 23, no. 33, pp. 4067-4078, 2013.

[15] S. Zhang, Y. Zhang, J. Liu et al., “Thiol modified $\mathrm{Fe}_{3} \mathrm{O}_{4} @ \mathrm{SiO}_{2}$ as a robust, high effective, and recycling magnetic sorbent for mercury removal," Chemical Engineering Journal, vol. 226, pp. 30-38, 2013.

[16] M. A. Ghasemzadeh, M. H. Abdollahi-Basir, and M. Babaei, " $\mathrm{Fe}_{3} \mathrm{O}_{4} @ \mathrm{SiO}_{2}-\mathrm{NH}_{2}$ core-shell nanocomposite as an efficient and green catalyst for the multi-component synthesis of highly substituted chromeno[2,3-b]pyridines in aqueous ethanol media," Green Chemistry Letters and Reviews, vol. 8, no. 3-4, pp. 40-49, 2015.

[17] S. H. Van Rijt, D. A. Bölükbas, C. Argyo et al., "Applicability of avidin protein coated mesoporous silica nanoparticles as drug carriers in the lung," Nanoscale, vol. 8, no. 15, pp. 8058-8069, 2016.

[18] C. Argyo, V. Weiss, C. Bräuchle, and T. Bein, "Multifunctional mesoporous silica nanoparticles as a universal platform for drug delivery," Chemistry of Materials, vol. 26, no. 1, pp. 435-451, 2013.

[19] C. Ayala-Orozco, J. G. Liu, M. W. Knight et al., "Fluorescence enhancement of molecules inside a gold nanomatryoshka," Nano Letters, vol. 14, no. 5, pp. 2926-2933, 2014.

[20] K. W. Shah, T. Sreethawong, S.-H. Liu, S.-Y. Zhang, L. S. Tan, and M.-Y. Han, "Aqueous route to facile, efficient and functional silica coating of metal nanoparticles at room temperature," Nanoscale, vol. 6, no. 19, pp. 11273-11281, 2014.

[21] Y. Huang, S. Xu, and V. S.-Y. Lin, "Bifunctionalized mesoporous materials with site-separated brønsted acids and bases: catalyst for a two-step reaction sequence," Angewandte Chemie International Edition, vol. 50, no. 3, pp. 661-664, 2011.

[22] J. Mosquera, I. García, M. Henriksen-Lacey, G. GonzálezRubio, and L. M. Liz-Marzán, "Reducing protein corona formation and enhancing colloidal stability of gold 
nanoparticles by capping with silica monolayers," Chemistry of Materials, vol. 31, no. 1, pp. 57-61, 2018.

[23] L. Shen, S. Pan, D. Niu et al., "Facile synthesis of organosilicacapped mesoporous silica nanocarriers with selective redoxtriggered drug release properties for safe tumor chemotherapy," Biomaterials Science, vol. 7, no. 5, pp. 1825-1832, 2019.

[24] S.-H. Cheng, W.-N. Liao, L.-M. Chen, and C.-H. Lee, "pHcontrollable release using functionalized mesoporous silica nanoparticles as an oral drug delivery system," Journal of Materials Chemistry, vol. 21, no. 20, pp. 7130-7137, 2011.

[25] A. Hakeem, F. Zahid, R. Duan et al., "Cellulose conjugated FITC-labelled mesoporous silica nanoparticles: intracellular accumulation and stimuli responsive doxorubicin release," Nanoscale, vol. 8, no. 9, pp. 5089-5097, 2016.

[26] Y. Wang, Y. Sun, J. Wang et al., "Charge-reversal aptesmodified mesoporous silica nanoparticles with high drug loading and release controllability," ACS Applied Materials \& Interfaces, vol. 8, no. 27, pp. 17166-17175, 2016.

[27] X. Chen, X. Cheng, A. H. Soeriyadi et al., "Stimuli-responsive functionalized mesoporous silica nanoparticles for drug release in response to various biological stimuli," Biomaterials Science, vol. 2, no. 1, pp. 121-130, 2014.

[28] D. Shao, X. Zhang, W. Liu et al., "Janus silver-mesoporous silica nanocarriers for SERS traceable and $\mathrm{pH}$-sensitive drug delivery in cancer therapy," ACS Applied Materials \& Interfaces, vol. 8, no. 7, pp. 4303-4308, 2016.

[29] Z. Wang, D. Shao, Z. Chang et al., "Janus gold nanoplatform for synergetic chemoradiotherapy and computed tomography imaging of hepatocellular carcinoma," ACS Nano, vol. 11, no. 12, pp. 12732-12741, 2017.

[30] D. Shao, J. Li, X. Zheng et al., "Janus "nano-bullets" for magnetic targeting liver cancer chemotherapy," Biomaterials, vol. 100, pp. 118-133, 2016.

[31] Z. Wang, F. Zhang, D. Shao et al., "Janus nanobullets combine photodynamic therapy and magnetic hyperthermia to potentiate synergetic anti-metastatic immunotherapy," Advanced Science, vol. 6, no. 22, Article ID 1901690, 2019.

[32] D. Shao, M. Li, Z. Wang et al., "Bioinspired diselenide-bridged mesoporous silica nanoparticles for dual-responsive protein delivery," Advanced Materials, vol. 30, no. 29, p. 1801198, 2018.

[33] W. Cheng, J. Nie, N. Gao et al., "A multifunctional nanoplatform against multidrug resistant cancer: merging the best of targeted chemo/gene/photothermal therapy," Advanced Functional Materials, vol. 27, no. 45, Article ID 1704135, 2017.

[34] W. Cheng, C. Liang, L. Xu et al., "TPGS-functionalized polydopamine-modified mesoporous silica as drug nanocarriers for enhanced lung cancer chemotherapy against multidrug resistance," Small, vol. 13, no. 29, Article ID 1700623, 2017.

[35] W. Gao, Y. Hu, L. Xu, M. Liu, H. Wu, and B. He, "Dual pH and glucose sensitive gel gated mesoporous silica nanoparticles for drug delivery," Chinese Chemical Letters, vol. 29, no. 12, pp. 1795-1798, 2018.

[36] C. Liang, H. Wang, M. Zhang et al., "Self-controlled release of oxaliplatin prodrug from $\mathrm{d}$ - $\alpha$-tocopheryl polyethylene glycol 1000 succinate (TPGS) functionalized mesoporous silica nanoparticles for cancer therapy," Journal of Colloid and Interface Science, vol. 525, pp. 1-10, 2018.

[37] X. Zeng, G. Liu, W. Tao et al., "A drug-self-gated mesoporous antitumor nanoplatform based on $\mathrm{pH}$-sensitive dynamic covalent bond," Advanced Functional Materials, vol. 27, no. 11, Article ID 1605985, 2017.
[38] Z. Zhang, A. Runa, J. Wu, H. Zhang, X. Li, and Z. He, "Bioresponsive nanogated ensemble based on structureswitchable aptamer directed assembly and disassembly of gold nanoparticles from mesoporous silica supports," Chinese Chemical Letters, vol. 30, no. 3, pp. 779-782, 2019.

[39] D. Shao, Z. Wang, W.-f. Dong et al., "Facile synthesis of coreshell magnetic mesoporous silica nanoparticles for $\mathrm{pH}$-sensitive anticancer drug delivery," Chemical Biology \& Drug Design, vol. 86, no. 6, pp. 1548-1553, 2015.

[40] J. Yue, S. z. Luo, M. m. Lu, D. Shao, Z. Wang, and W. f. Dong, "A comparison of mesoporous silica nanoparticles and mesoporous organosilica nanoparticles as drug vehicles for cancer therapy," Chemical Biology \& Drug Design, vol. 92, no. 2, pp. 1435-1444, 2018.

[41] Z. Chen, L. Wan, Y. Yuan et al., "pH/GSH-dual-sensitive hollow mesoporous silica nanoparticle-based drug delivery system for targeted cancer therapy," ACS Biomaterials Science \& Engineering, vol. 6, no. 6, pp. 3375-3387, 2020.

[42] A. M. Alswieleh, M. M. Alshahrani, K. E. Alzahrani et al., "Surface modification of $\mathrm{pH}$-responsive poly(2-(tert-butylamino)ethyl methacrylate) brushes grafted on mesoporous silica nanoparticles," Designed Monomers and Polymers, vol. 22, no. 1, pp. 226-235, 2019. 